\title{
The pattern of IL-24/mda-7 and its cognate receptors expression following activation of human hepatic stellate cells
}

\author{
IMAN JAMHIRI $^{1}$, SEYED YOUNES HOSSEINI ${ }^{2}$, DAVOOD MEHRABANI ${ }^{3}$, \\ ZAHRA KHODABANDEH $^{3}$, RAMIN YAGHOBI ${ }^{4}$, RAZIEH DOWRAN ${ }^{2}$ and SABER ZAHRI ${ }^{1}$ \\ ${ }^{1}$ Department of Biology, Cell and Molecular Laboratory, Faculty of Science, University of Mohaghegh Ardabili, \\ Ardabil 56199-11367; ${ }^{2}$ Department of Bacteriology and Virology; ${ }^{3}$ Stem Cell Technology Research Center; \\ ${ }^{4}$ Transplant Research Center, Shiraz University of Medical Sciences, Shiraz 71348-14336, Iran
}

Received March 10, 2017; Accepted June 6, 2017

DOI: $10.3892 /$ br.2017.931

\begin{abstract}
Activation of hepatic stellate cells (HSCs) is the pivotal event during liver fibrosis. Interleukin (IL)-24/melanoma differentiation-associated gene-7 (mda-7) has attracted attention in the pathophysiology of some diseases, while its role in activation/suppression of human HSCs is still unclear. It is important to elucidate whether the expression levels of the IL-24/mda-7 protein and its receptors in HSC cells are changed following activation. LX-2 cells, a human hepatic stellate cell line were activated by a combination of leptin and serum starvation. The activation state was evaluated through measuring the mRNA expression of profibrotic molecules, collagen-I, TIMP metalloproteinase inhibitor-1 and transforming growth factor- $\beta$. The expression of IL- $24 / \mathrm{mda}-7$ was assessed in mRNA and protein levels by reverse transcription-quantitative polymerase chain reaction (RT-qPCR) and ELISA methods, respectively. Hence, the amount of IL-22R1 and IL-20R2 subunit expression was also compared in activated and normal LX-2 cells by RT-qPCR. The expression level of IL-24/mda-7 and its cognate receptors was detectable both in the normal and activated LX-2 cell line. Furthermore, in activated LX-2, a significant increase of IL24 expression either on IL-22R1 and IL-20R2 subunits was also noticeable in comparison to normal cells. The activation state of LX-2 cells caused significant changes of IL-24/mda-7 and its receptors expression. In addition, the elevation in IL-24/mda-7 during LX-2 cell activation, suggested that IL-24/mda-7 and its cognate receptors serve a possible role in the development of the fibrosis process. Therefore, IL-24/mda-7 and relevant signaling pathways may be employed as a target for fibrosis treatment.
\end{abstract}

Correspondence to: Dr Seyed Younes Hosseini, Department of Bacteriology and Virology, Shiraz University of Medical Sciences, Shiraz 71348-14336, Iran

E-mail: hoseiniy@sums.ac.ir

Key words: human hepatic stellate cells, LX-2, IL-24/mda-7, IL-20R2, IL-22RI

\section{Introduction}

Persistent liver injury resembles the chronic wound healing response, which consequently leads to liver fibrosis. Liver fibrosis is defined as an excessive accumulation of extracellular matrix (ECM) proteins in the tissue arising in a wide spectrum of disorders including viral hepatitis, metabolic and alcohol consumption related diseases $(1,2)$. In spite of different possible mechanisms behind the establishment of the liver fibrosis, some dimension of phenomena needs to be defined more.

Among liver resident cells, hepatic stellate cells (HSCs) have a profound role in fibrosis development, which is demonstrated by ECM production and deposition. The persistence activation of HSCs following tissue injury is attributed as the most essential event underlying liver fibrogenesis (3). During the fibrosis progress, quiescent HSCs transform into myofibroblast-like cells, which acquire modified phenotype with plentiful secretion of ECM proteins (4).

Leptin, a 16-kDa hormone has direct effects on angiogenesis, immunity and hematopoiesis as well as the metabolism of liver cells. This protein has been employed by numerous groups for experimental liver fibrogenesis (5). In vitro fibrosis studies demonstrated that serum starvation and leptin treatment together are sufficient to activate HSC model cells, LX-2, due to significant elevated ECM and $\alpha$-smooth muscle actin level in culture $(6,7)$. Moreover, several studies have shown that leptin serves an important role in inflammation and related pathogenesis in liver particularly in fibrosis process. The plasma level of leptin has been reported to be increased in cirrhosis and steatohepatitis suffering patients $(8,9)$. The results suggested leptin as a reliable fibrosis induction molecule with the propensity of inflammation establishment $(5,10)$.

Interleukin (IL)-24/melanoma differentiation associated gene (mda)-7 is a member of the IL-10 cytokine family that performs a unique antitumor activity. Historically, mda-7 was discovered using the subtraction hybridization method on cDNA libraries from melanoma cells, that its reduced expression was significant. $(11,12)$. It serves an important role in fibroproliferative diseases, including pulmonary fibrosis, chronic kidney diseases, inflammatory bowel diseases and cardiovascular diseases (13). 
The cytokine exhibits different site-dependent activities regardless of its family common properties. Whereas the pro-inflammatory role is almost supposed as the main function of IL-24/mda7, the anti-proliferative features and differentiation induction impact was also assigned to it. IL-24/mda-7 is expressed mainly in cells like NK cells, melanocytes, B cells, dendritic cells and monocytes (14).

The cytokine has two heterodimeric receptors, IL-22R1/IL-20R2 and IL-20R1/IL-20R2, which, like other cytokines, is triggered through the Jak-STAT pathway (15). As IL-24/mda-7 exhibits characteristics of apoptosis induction in transforming cells, its utility for cancer gene therapy approaches in heavy is considered by several groups $(16,17)$. Furthermore, a recent study has indicated that the IL-10 cytokine family can be effective in ECM production with a pivotal role in fibrosis, but the underlying mechanism by which IL-24/mda-7 may inhibit ECM production, is still unknown (13).

In spite of studies regarding the role of IL-24/mda-7 in psoriasis and inflammatory bowel diseases, the possible role in other diseases needs to be investigated. As an inflammatory-related disease, fibrosis involved cells may also be affected by the expression level of this cytokine. So it is noteworthy to clarify if the expression levels of IL-24/mda-7 protein and cognate receptors in HSC cells are changed following activation. The aims of the study were to assess whether the fibrogenic actions of leptin leaves significant changes in IL-24/mda-7 secretion and cognate receptor expression in the LX-2 cell line, a human-derived HSCs.

\section{Materials and methods}

Cell culture. LX-2, an immortalized human HSC line, was a gift provided by Professor Friedman (Mount Sinai School of Medicine, New York, NY, USA). All the details of the generation of this unique line have been described previously (18). The cell line was cultured in Dulbecco's modified Eagle's medium (DMEM) complemented with $5 \%$ fetal bovine serum (FBS), $1 \%$ non-essential amino acids, $100 \mathrm{U} / \mathrm{ml}$ penicillin and $100 \mathrm{mg} / \mathrm{ml}$ streptomycin. Cells were distributed in six-well plates in 200,000 cells/well and treatment was performed when their confluence reached $80 \%$. They were incubated under humidified standard condition, at $37^{\circ} \mathrm{C}$ and $5 \% \mathrm{CO}_{2}$. The activation procedure, leptin addition and serum starvation concomitant were applied on LX-2 cells for $24 \mathrm{~h}$. For serum starvation conditions, 1\% DMEM was employed instead of 5\% DMEM. The purified Leptin was used at concentrations of $25-100 \mathrm{ng} / \mathrm{ml}$.

Real-time PCR analysis. Total cellular RNA was isolated from inactive LX-2 as control and active LX-2 (treated with leptin) by RNA extraction kit (CinnaGen Inc., Tehran, Iran). The quantity and quality of obtained RNA were checked by measuring the ratio of optical density of 260/280 nm using Nanodrop $^{\text {TII }}$ spectrophotometer (Nanodrop; Thermo Fisher Scientific, Wilmington, DE, USA) and then was stored at $-80^{\circ} \mathrm{C}$ until cDNA synthesis. The cDNA was then synthesized using 1,000 ng total RNA in a first-strand cDNA synthesis reaction by the help of RevertAid ${ }^{\mathrm{TN}}$ First Strand cDNA Synthesis kit (Thermo Fisher Scientific, Inc., Waltham, MA, USA). Reverse transcription-quantitative polymerase chain reaction (RT-qPCR) was performed using the ABI Biosystems StepOne and the RealQ Plus 2x Master Mix Green (Ampliqon $\mathrm{A} / \mathrm{S}$, Odense, Denmark). In each reaction, $200 \mathrm{nM}$ of each primer (Table I) was added to target the specific sequence. Specific primers targeting IL-24/mda7, IL-20R2, IL-22R1, collagen (Col)-I, TIMP metalloproteinase inhibitor-1 (TIMP-1) and transforming growth factor (TGF)- $\beta$ were designed as shown in Table I. The PGK housekeeping gene was also used as internal control of qPCR reactions. The qPCR conditions were set for $10 \mathrm{~min}$ at $94^{\circ} \mathrm{C}$ followed by 40 cycles of $15 \mathrm{sec}$ at $94^{\circ} \mathrm{C}, 60 \mathrm{sec}$ at $58^{\circ} \mathrm{C}$ and final extension of $7 \mathrm{~min}$ at $72^{\circ} \mathrm{C}$. The amplification signals of different samples were normalized to PGK cycle threshold $(\mathrm{Ct})$, and then $2^{-\Delta \Delta \mathrm{Cq}}$ method was applied for comparing mRNA levels of activated vs. the control (normal LX-2), which represented as fold-change in data analysis (19).

ELISA. At $\sim 48 \mathrm{~h}$ following LX-2 cell activation with leptin and serum starvation, the culture supernatants were accumulated for measurement of the released TGF- $\beta$ by a human-mouse TGF- $\beta$ ELISA kit (eBioscience Inc., San Diego, CA, USA,) according to the manufacturer's instructions. The final absorbance was read at $450 \mathrm{~nm}$ by the ELISA reader with using a FLUOstar OMEGA microplate reader and software (BMG Labtech Ltd., Aylesbury, UK). and the concentration of TGF- $\beta$ protein was evaluated comparing with a standard curve.

In addition, the culture supernatants from the leptin-treated and untreated LX-2 cells were collected following 24 and $48 \mathrm{~h}$ of incubation. Then they were evaluated using an ELISA kit, IL-24/mda-7 human ELISA kit (Abcam, Cambridge, MA, USA; cat. no. ab171345). The final absorbance was read at $450 \mathrm{~nm}$ and the concentration of IL-24/mda-7 protein was evaluated comparing with a standard curve.

Statistical analysis. Data were expressed as the mean \pm standard error of the mean and were analyzed by independent samples t-test, using GraphPad Prism 6 (GraphPad Software, Inc., La Jolla, CA, USA). P $<0.05$ was considered to indicate a statistically significant difference between group means.

\section{Results}

Leptin treatment activates LX-2 fibrotic phenotype. To complete the activation process of LX-2, leptin treatment, concomitant with serum starvation was applied on culture media. Leptin treatment with serum starvation could induce conversion of LX-2 cells to activate a phenotype characterized by veiled morphology in the culture plate (20) and expression of pro-fibrogenic genes, including Col-I, TIMP-1 and TGF- $\beta$.

The expression analysis of pro-fibrotic genes indicated that, in activated LX-2, the expression pattern of TGF- $\beta(\mathrm{P}=0.0061)$, Col-I $(\mathrm{P}=0.012)$ and TIMP-1 $(\mathrm{P}=0.0047)$ were significantly higher than normal cells, as depicted in Fig. 1. The ELISA results were also in agreement with gene expression, as the concentration of TGF- $\beta$ protein in the culture supernatant was significantly higher than in normal cells $(\mathrm{P}<0.0001)$. The TGF- $\beta$ protein concentrations were determined near to $382.5 \mathrm{pg} / \mathrm{ml}, 237 \mathrm{pg} / \mathrm{ml}$ for $100 \mathrm{ng}$ and $25 \mathrm{ng}$ leptin treatments, respectively, in comparison to $30.5 \mathrm{pg} / \mathrm{ml}$ for normal cells (Fig. 1). 
Expression of IL-24/mda-7 in LX-2 cells. When the expression pattern of IL-24/mda-7 was evaluated in mRNA and protein levels, it revealed that LX-2 cells normally expressed this protein as detected by RT-qPCR and ELISA methods. It was also identified that activation phenotype was associated with a two-folds increment in IL-24/mda-7 mRNA when compared to normal cells $(\mathrm{P}=0.0002)$. The ELISA results were also in agreement with gene expression as the concentration of IL-24/mda-7 protein in culture supernatant was significantly higher than normal cells $(\mathrm{P}<0.01)$. The IL-24/mda-7 protein concentration was determined near to $961.1 \mathrm{pg} / \mathrm{ml}, 918.1 \mathrm{pg} / \mathrm{ml}$ and $438.5 \mathrm{pg} / \mathrm{ml}$ for 24 and $48 \mathrm{~h}$ treatments and for normal cells, respectively (Fig. 2).

Expression of IL-24/mda-7 receptors in LX-2 cell lines. When the expression level of two different IL-24/mda-7 receptors mRNAs (IL-20R1 and IL-22R1) were monitored through RT-qPCR, these were demonstrated that in normal cells, both receptors were expressed at least in $\mathrm{mRNA}$ level. Hence, in activated LX-2, a significant elevation of IL-20R1 $(\mathrm{P}<0.0001)$ and IL-22R1 receptors with more than $>2$-fold changes $(\mathrm{P}=0.0079)$ were detectable when compared to normal cells. While the expression changes of both receptors were similar, IL-20R2 elicited more than IL-22R following activation process. These results suggested that stress condition (leptin and serum starvation) as expected, lead to a significant increase in the expression pattern of both investigated IL-24/mda-7 receptors (Fig. 3).

\section{Discussion}

The pathophysiology of fibrosis as a durable response remained unresolved in some fields. The possible role of IL-10 family members such as IL-20, IL-22 and, more recently, IL-24/mda-7, started to be delineated more in fibrosis development (13). Even though the general player molecules involved in HSC cell activation and transition into myofibroblasts have been studied deeply, the possible role of IL-24/mda7 needs to be investigated in liver fibrosis. Based on the authors' knowledge, this is the first report considering IL-24/mda-7 importance in HSC activation.

The increased expression of IL-24/mda-7 in relevance to the renovation of the kidney was recently reported (21). As mentioned, liver injury is similar to other chronic wound healing responses, which consequently leads to liver fibrosis. In this context, the aims of the present study were to evaluate whether IL-24/mda-7 and cognate receptor (IL-22R1 and IL-20R2) subunits were expressed in LX2 cells and what will happen to their expression level in response to in vitro activation. The results indicated that stress conditions encompassing leptin treatment and starvation induce LX2 cell activation, confirmed by the higher expression of TGF- $\beta$, TIMP- 1 and collagen profibrotic genes as described in a previous study (22). The data were correlated with previous reports that claimed leptin to prompt fibrosis signaling pathways $(6,23)$. The results demonstrated that the LX-2 cells have been activated.

Based on the first findings, it was shown that IL-24/mda7 was expressed in measurable levels even before activation, as mRNA and protein were detectable by RT-qPCR and ELISA. Besides, it was indicated that, in activated cells, IL-24/mda-7 expression was significantly noticeable compared to baseline expression of normal LX-2. The expression levels of IL-24 in activated LX-2 cells are steady state around 24 and $48 \mathrm{~h}$. This data correlated with the increases seen in hepatic IL-22 mRNA levels. IL-22 has an important role in the liver regeneration and this cytokine has functional similarities to IL-24/mda-7 (24,25).

The reports considering IL-24/mda-7 expression in human HSCs are limited. Although, expression level of IL-20 and IL-22 were evaluated in human and mice stellate cells, comparing IL-24/mda-7 expression in normal or activated HSCs is rare $(13,26)$. New research revealed that IL-22, another member of the IL-10 cytokine family, promotes HSC proliferation and activation, prevents HSC apoptosis, induces the overexpression of HSC growth factors and fibrosis markers including Col-IV, laminin and hyaluronic acid (27). But unlike IL-22, no definite role in liver fibrosis was assigned for IL-24/mda-7, in spite of various studies that have reported its anticancer properties $(24,28)$.

It must be noted that LX-2 is accepted as a semi-activated cell, so the level of IL-24/mda-7 in the current experiments may not independently reflect the state of completely normal human stellate cells. Previously, Imaeda et al (26) detected a significant level of expression for IL-24/mda-7 in pancreatic stellate cells. They supposed that this cytokine may have a role in the pathology of chronic pancreatitis cases. Whereas the exact mechanism responsible for IL-24/mda-7 expression is not described before, an involvement of MAPK pathway and STAT3 activation is under consideration $(29,30)$.

It is reported that IL-24/mda-7 is expressed by various cell types including B cells, dendritic cells, natural killer cells, melanocytes and monocytes (31). Despite the ambiguous role in immunity, increased expression severely correlates with differentiation state of the cells. Regarding TGF- $\beta$ in fibrosis development, it may be proposed that IL-24/mda-7 acts in a similar manner. In other words, an increase in IL-24/mda-7 expression may be a controlling feedback in early fibrosis that thereafter exacerbate the responsible mechanisms (32). Conversely, to compare with other cell lines such as HepG2, it was indicated that IL-24/mda-7 has higher expression in the LX-2 cell line, which reveals the important role of this protein in activation of LX-2 cell line (33). The mouse IL-24/mda-7-like molecule, termed FISP (IL-4-induced secreted protein) and the rat IL-24/mda-7-like molecule, termed C49A, increased in wound repair (34). This observation led to the hypothesis that expression of IL-24/mda-7 in vivo may exhibit impact on the fibrogenesis process. However, some other properties are not similar among species indicating their principle differences. It may be predicted that the investigation of IL-24/mda7 in other species may mislead scientists. It should be noted that evaluation of fibrotic gene expression for mediating treatment responses to IL-24/mda-7 will be the subject of ongoing study in animal models. Also, assessment of the expression level for this protein and cognate receptors should be performed by other methods such as western blotting, immunohistochemistry or immunoblotting, a limitation of the current study.

In order to perform its autocrine function, IL-24/mda-7 binds and signals through the heterodimeric IL-22R1/IL-20R2 and IL-20R1/IL-20R2 receptors, following by STAT-3 phosphorylation. The effectiveness of IL-24/mda-7 is 
Table I. Primers used in the present study.

\begin{tabular}{llc}
\hline Gene name & \multicolumn{1}{c}{ Primer Sequence $\left(5^{\prime}-3^{\prime}\right)$} & Size $(\mathrm{bp})$ \\
\hline IL-24/mda-7 & Forward: TGTGAAAGACACTATGCAAGCTC & 224 \\
IL-20R2 & Reverse: GTGACACGATGAGAACAAAGTTG & 138 \\
& Forward: AGGCCCAGACATTCGTGAAG \\
Reverse: CGACCACAAGGATCAGCATGA & 181 \\
& Forward: TGCTGACCATCTTGACTGTG \\
TGF- $\beta$ & Reverse: TCCCTCTCTCCGTACGTCTTAT & 136 \\
Col-I & Forward: AAGGCGAAAGCCCTCAATTT \\
& Reverse: CAGCAACAATTCCTGGCGATA & 140 \\
TIMP-1 & Forward: GAGGGCCAAGACGAAGACATC \\
& Reverse: CAGATCACGTCATCGCACAAC \\
PGK & Forward: TACTTCCACAGGTCCCACAAC & 202 \\
& Reverse: GTTTGCAGGGGATGGATAAAC & 116 \\
& Forward: TAAAGCCGAGCCAGCCAAAA & \\
& Reverse: CTCCTACCATGGAGCTGTGG &
\end{tabular}

bp, base pair; IL, interleukin; mda, melanoma differentiation associated gene; TGF- $\beta$, transforming growth factor- $\beta$; Col-I, collagen I; TIMP-1, TIMP metalloproteinase inhibitor-1.
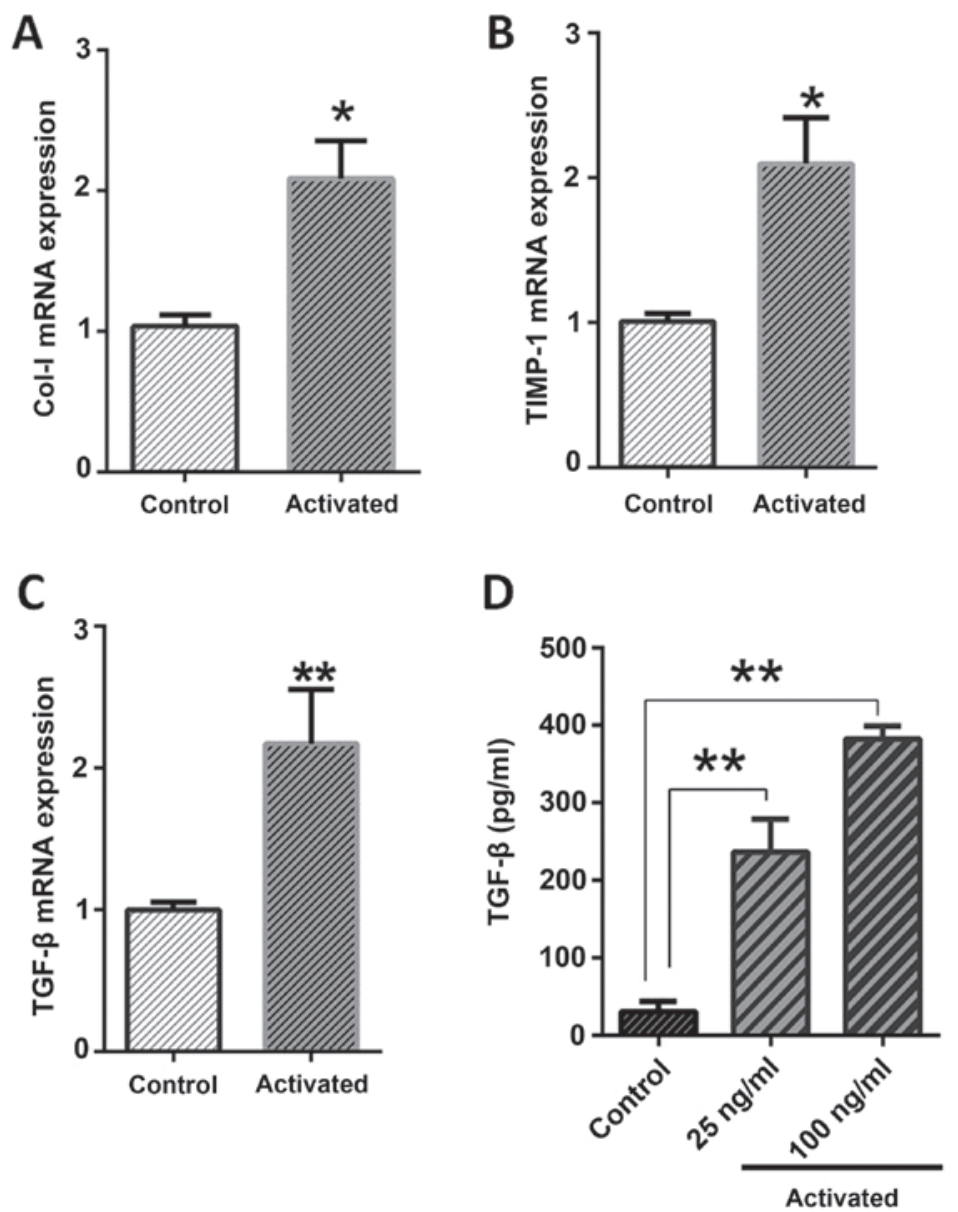

Figure 1. Col-I, TIMP-1 and TGF- $\beta$ expression changes in normal and activated cells. Control LX-2 cell received 5\% Dulbecco's modified Eagle's medium, FBS and activated LX-2 cell treatment with leptin/serum starvation. (A) Stress condition effect on Col-I expression in LX-2 cells. The cell Col-I mRNA expression was analyzed by reverse transcription-quantitative polymerase chain reaction following $24 \mathrm{~h}$. (B) Stress condition effect on TIMP-1 expression in LX-2 cells. The cell TIMP-1 mRNA expression was analyzed by reverse transcription-quantitative polymerase chain reaction following $24 \mathrm{~h}$. (C) mRNA expression of TGF- $\beta$ in normal and activated cells was analyzed by reverse transcription-quantitative polymerase chain reaction following 24 h. (D) An increase in the amount of TGF- $\beta$ protein by ELISA on serum starvation and $25 \mathrm{ng} / 100 \mathrm{ng}$ leptin treatment was noticed. Data (mean \pm standard error of the mean) are shown. Each column is representative of group mean (mean \pm standard error of the mean). ${ }^{*} \mathrm{P}<0.05,{ }^{* * *} \mathrm{P}<0.01$ vs. control. Col-I, collagen-I; TIMP-1, TIMP metalloproteinase inhibitor-1; TGF- $\beta$, transforming growth factor- $\beta$. 
A

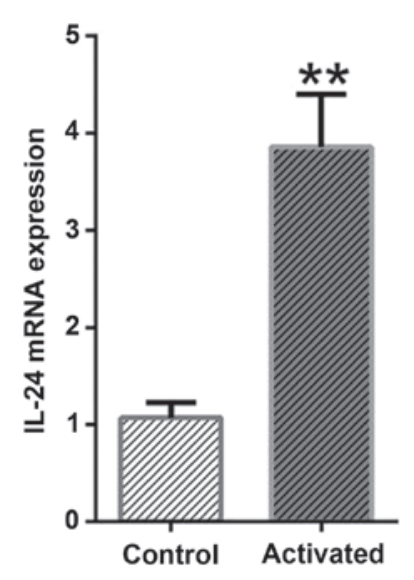

B

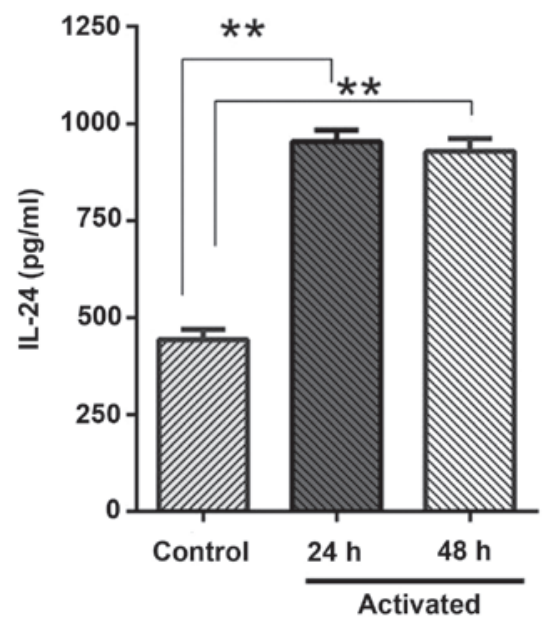

Figure 2. Stress condition effect on the expression of IL-24/mda-7 in LX-2 cells. (A) Serum starvation and leptin treatment increase IL-24/mda-7 mRNA expression level. The mRNA expression of IL-24/mda-7 in normal and activated cell was analyzed by reverse transcription-quantitative polymerase chain reaction on $24 \mathrm{~h}$. (B) An increase in the amount of IL-24/mda-7 protein by ELISA on 24 and $48 \mathrm{~h}$ following stress condition was observed. Data (mean \pm standard error of the mean) are shown. The analysis of ELISA and reverse transcription-quantitative polymerase chain reaction demonstrated that leptin and serum starvation obviously increased the expression of IL-24/mda-7. ${ }^{*} \mathrm{P}<0.05,{ }^{* *} \mathrm{P}<0.01$ vs. control. IL, interleukin; mda, melanoma differentiation associated gene.

A

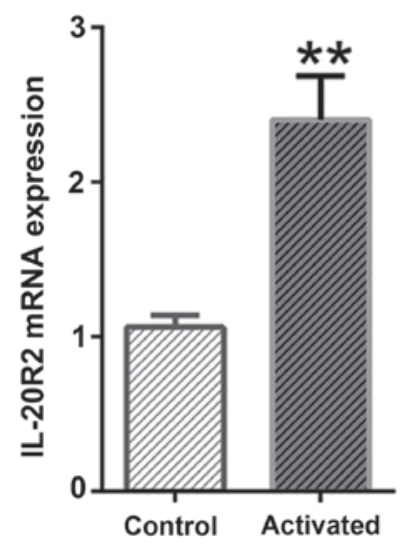

B

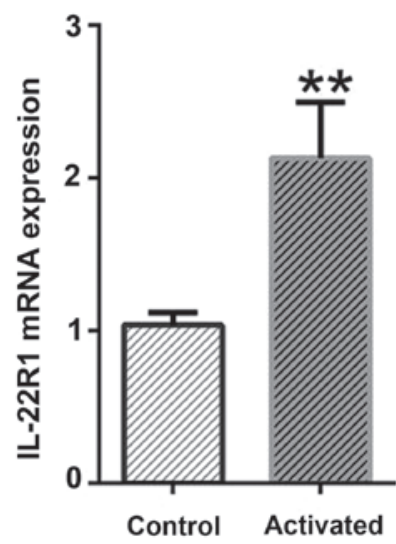

Figure 3. IL-24/mda-7 receptors fold-change expression in normal and active LX-2 cells. (A) Stress condition effect on IL-20R2 expression in LX-2 cells. The cells were IL-20R mRNA expression was sequentially determined by reverse transcription-quantitative polymerase chain reaction. (B) Stress condition effect on IL-22R1 expression in LX-2 cells. The IL-22R1 expression significantly increased in activated LX-2 cells. Gene expression of IL-24/mda-7 receptors was analyzed by reverse transcription-quantitative polymerase chain reaction at $24 \mathrm{~h}$ following stress conditions. Expression data relative to those of the housekeeping gene from at least three independent assays are given as mean \pm standard error of the mean. Statistical significance was tested using the independent samples t-test. $\mathrm{P}<0.05,{ }^{* *} \mathrm{P}<0.01$ vs. control. IL, interleukin; mda, melanoma differentiation associated gene.

dependent on the presence of these receptors on target cells. The finding revealed that IL-20R2 and IL-22R1 receptors were not only expressed on the LX-2 cells, but were also upregulated following cell activation. One of the reasons that the authors focused on IL-22R1/IL-20R2 instead of the other heterodimers is because of the interaction between IL-22R1/IL-20R2 and IL-24 receptor complexes is a key mediator for the wound healing response, a process that mimics fibrosis in the liver (35).

In addition, Imaeda et al demonstrated that these receptors were detectable in chronic pancreatitis tissue of suffering patients (26). The expression of IL-20R2 and IL-22R1 was also investigated elsewhere, and IL-22R1 expression was reported to be distributed among the kidney, liver, pancreas and skin (13).
Furthermore, in another study, it was revealed that IL-20R1 and IL-20R2 were expressed in synovial cells (RASCs), and the results indicated that RASCs had constitutive expression of IL-20R1 and IL-20R2 (36). Furthermore, Kunz et al identified that IL-20R2 expression levels in keratinocytes increased followed by STAT3 activation (30). Conversely, Sarkar et al (34) analyzed 12 mycoplasma-free melanoma cell lines finding that lack IL-24/mda-7 receptors in some mycoplasma-free melanoma cell lines disrupted Jak/STAT pathway. However, the expression of IL-24/mda-7 receptors in a liver cell line, such as HepG2, is still not well understood. Results of the current study have provided evidence that IL-22RA1 and IL-20R2 were consistently expressed in active and normal LX-2 cell lines. In future study, more reliable results can be achieved by analyzing IL22R1/IL-20R2 protein expression with western blotting. 
In conclusion, expression of IL-24/mda-7 and its cognate receptors in the normal LX-2 cell line emphasized their possible impact on fibrogenesis process. In addition, leptin could increase the expression of IL-24/mda-7 and its receptors implying that IL-24/mda-7 ought to be investigated more as a new target for fibrosis therapy. The unraveling of the unknown mechanism underling IL-24/mda-7 function during fibrosis may also be useful to understand the pathology of the disease and to open a path for new therapeutic drugs.

\section{References}

1. Rockey DC: Current and future anti-fibrotic therapies for chronic liver disease. Clin Liver Dis 12: 939-962, 2008.

2. Bataller R and Brenner DA: Liver fibrosis. J Clin Invest 115: 209-218, 2005.

3. Kisseleva T and Brenner DA: Role of hepatic stellate cells in fibrogenesis and the reversal of fibrosis. J Gastroenterol Hepatol 22 (Suppl 1): S73-S78, 2007.

4. Jiang JX and Török NJ: Liver injury and the activation of the hepatic myofibroblasts. Curr Pathobiol Rep 1: 215-223, 2013.

5. Loffreda S, Yang SQ, Lin HZ, Karp CL, Brengman ML, Wang DJ, Klein AS, Bulkley GB, Bao C, Noble PW, et al: Leptin regulates proinflammatory immune responses. FASEB J 12: 57-65, 1998.

6. Cao Q, Mak KM and Lieber CS: Leptin represses matrix metalloproteinase-1 gene expression in LX2 human hepatic stellate cells. J Hepatol 46: 124-133, 2007.

7. Yegorov YE, Akimov SS, Hass R, Zelenin AV and Prudovsky IA: Endogenous beta-galactosidase activity in continuously nonproliferating cells. Exp Cell Res 243: 207-211, 1998.

8. Uygun A, Kadayifci A, Yesilova Z, Erdil A, Yaman H, Saka M, Deveci MS, Bagci S, Gulsen M, Karaeren N and Dagalp K: Serum leptin levels in patients with nonalcoholic steatohepatitis. Am J Gastroenterol 95: 3584-3589, 2000.

9. Saxena NK, Saliba G, Floyd JJ and Anania FA: Leptin induces increased alpha2(I) collagen gene expression in cultured rat hepatic stellate cells. J Cell Biochem 89: 311-320, 2003.

10. Crespo J, Rivero M, Fábrega E, Cayón A, Amado JA, García-Unzeta MT and Pons-Romero F: Plasma leptin and TNF-alpha levels in chronic hepatitis $\mathrm{C}$ patients and their relationship to hepatic fibrosis. Dig Dis Sci 47: 1604-1610, 2002.

11. Pestka S, Krause CD, Sarkar D, Walter MR, Shi Y and Fisher PB: Interleukin-10 and related cytokines and receptors. Annu Rev Immunol 22: 929-979, 2004.

12. Jiang H, Lin JJ, Su ZZ, Goldstein NI and Fisher PB: Subtraction hybridization identifies a novel melanoma differentiation associated gene, mda-7, modulated during human melanoma differentiation, growth and progression. Oncogene 11: 2477-2486, 1995.

13. Sziksz E, Pap D, Lippai R, Béres NJ, Fekete A, Szabó AJ and Vannay A: Fibrosis related inflammatory mediators: Role of the IL-10 cytokine family. Mediators Inflamm 2015: 764641, 2015.

14. Patani N, Douglas Jones A, Mansel R, Jiang W and Mokbel K Tumour suppressor function of MDA 7/IL 24 in human breast cancer. Cancer Cell Int 10: 29, 2010.

15. Wang M, Tan Z, Zhang R, Kotenko SV and Liang P: Interleukin 24 (MDA-7/MOB-5) signals through two heterodimeric receptors, IL-22R1/IL-20R2 and IL-20R1/IL-20R2. J Biol Chem 277: 7341-7347, 2002.

16. Huang EY, Madireddi MT, Gopalkrishnan RV, Leszczyniecka M, Su Z, Lebedeva IV, Kang D, Jiang H, Lin JJ, Alexandre D, et al: Genomic structure, chromosomal localization and expression profile of a novel melanoma differentiation associated (mda-7) gene with cancer specific growth suppressing and apoptosis inducing properties. Oncogene 20: 7051-7063, 2001.

17. Hosseini E, Hosseini SY, Hashempour T, Fattahi MR and Sadeghizadeh M: Effect of RGD coupled MDA-7/IL-24 on apoptosis induction in a hepatocellular carcinoma cell line. Mol Med Rep 15: 495-501, 2017.

18. Yang C, Zeisberg M, Mosterman B, Sudhakar A, Yerramalla U, Holthaus K, Xu L, Eng F, Afdhal N and Kalluri R: Liver fibrosis: Insights into migration of hepatic stellate cells in response to extracellular matrix and growth factors. Gastroenterology 124: 147-159, 2003.
19. Livak KJ and Schmittgen TD: Analysis of relative gene expression data using real time quantitative PCR and the $2^{-\Delta \Delta C q}$ method. Methods 25: 402-408, 2001.

20. Hosseini SY, Kalantar K, Shahin K, Ghayour M, Rajabibazl M, Fattahi MR, et al: Comparison of the in vitro antifibrogenic effects of silymarin, silybin A and $18 \alpha$ glycyrrhizin on activated hepatic stellate cells. Jundishapur J Nat Pharm Prod Inpress: e40285, 2016.

21. Pap D, Sziksz E, Kiss Z, Rokonay R, Veres-Székely A, Lippai R, Takács IM, Kis É, Fekete A, Reusz G, et al: Microarray analysis reveals increased expression of matrix metalloproteases and cytokines of interleukin-20 subfamily in the kidneys of neonate rats underwent unilateral ureteral obstruction: A potential role of IL-24 in the regulation of inflammation and tissue remodeling. Kidney Blood Press Res 42: 16-32, 2017.

22. Khanizadeh S, Ravanshad M, Hosseini S, Davoodian P, Nejati Zadeh A and Sarvari J: Blocking of SMAD4 expression by shRNA effectively inhibits fibrogenesis of human hepatic stellate cells. Gastroenterol Hepatol Bed Bench 8: 262-269, 2015.

23. Marra F: Leptin and liver fibrosis: A matter of fat. Gastroenterology 122: 1529-1532, 2002.

24. Tian H, Zhang DF, Zhang BF, Li HZ, Zhang Q, Li LT, Pei DS and Zheng JN: Melanoma differentiation associated gene-7/interleukin-24 induces caspase-3 denitrosylation to facilitate the activation of cancer cell apoptosis. J Interferon Cytokine Res 35: $157-167,2015$.

25. Ren X, Hu B and Colletti LM: IL-22 is involved in liver regeneration after hepatectomy. Am J Physiol Gastrointest Liver Physiol 289: G74-G80, 2009.

26. Imaeda $H$, Nishida $A$, Inatomi $O$, Fujiyama $Y$ and Andoh $A$ : Expression of interleukin-24 and its receptor in human pancreatic myofibroblasts. Int J Mol Med 28: 993-999, 2011.

27. Gao Y, Ren H, Meng F, Li J, Cheung E, Li H, Zhao J, Liu H, Liu $\mathrm{Z}$ and Zhang M: Pathological roles of interleukin-22 in the development of recurrent hepatitis $\mathrm{C}$ after liver transplantation. PLoS One 11: e0154419, 2016.

28. Hu CW, Yin GF, Wang XR, Ren BW, Zhang WG, Bai QL, Lv YM, Li WL and Zhao WQ: IL-24 induces apoptosis via upregulation of RNA-activated protein kinase and enhances temozolomide-induced apoptosis in glioma cells. Oncol Res 22: 159-165, 2014.

29. Shefler I, Pasmanik-Chor M, Kidron D, Mekori YA and Hershko AY: T cell-derived microvesicles induce mast cell production of IL-24: Relevance to inflammatory skin diseases. J Allergy Clin Immunol 133: 217-224, 2014.

30. Kunz S, Wolk K, Witte E, Witte K, Doecke WD, Volk HD, Sterry W, Asadullah K and Sabat R: Interleukin (IL)-19, IL-20 and IL-24 are produced by and act on keratinocytes and are distinct from classical ILs. Exp Dermatol 15: 991-1004, 2006.

31. Mirzaei MH and Esmaeilzadeh A: Overexpression of MDA-7/IL-24 as an anticancer cytokine in gene therapy of thyroid carcinoma. J Med Hypotheses Ideas 8: 7-13, 2014.

32. Jiang H, Su ZZ, Lin JJ, Goldstein NI, Young CS and Fisher PB: The melanoma differentiation associated gene mda-7 suppresses cancer cell growth. Proc Natl Acad Sci USA 93: 9160-9165, 1996.

33. Xiao CW, Xue XB, Zhang H, Gao W, Yu Y, Chen K, Zheng JW and Wang CJ: Oncolytic adenovirus-mediated MDA-7/IL-24 overexpression enhances antitumor activity in hepatocellular carcinoma cell lines. Hepatobiliary Pancreat Dis Int 9: 615-621, 2010.

34. Sarkar D, Su ZZ, Lebedeva IV, Sauane M, Gopalkrishnan RV, Dent P and Fisher PB: mda-7 (IL-24): Signaling and functional roles. Biotechniques 33: 30-39, 2002.

35. Kirsch S. The effect of cytokines of the Interleukin-10-family on cutaneous wound healing. Freie Universität Berlin, 2013 (In German).

36. Sakurai N, Kuroiwa T, Ikeuchi H, Hiramatsu N, Maeshima A, Kaneko Y, Hiromura K and Nojima Y: Expression of IL-19 and its receptors in RA: Potential role for synovial hyperplasia formation. Rheumatology (Oxford) 47: 815-820, 2008. 